\title{
Richter's syndrome developing in a patient with adult onset Still's disease
}

\author{
D. E. M. Geurts • W. J. F. M. van der Velden • \\ K. M. Hebeda • J. M. M. Raemaekers
}

Received: 24 December 2007 / Accepted: 3 June 2008 / Published online: 8 July 2008

(C) The Author(s) 2008

\section{Dear Editor,}

Autoimmune disorders can be a risk factor for the development of lymphoma [1], and at the same time, malignant lymphoma can present with paraneoplastic syndromes consisting of autoimmune phenomena and even full-blown rheumatoid disorders [2]. When a patient presents with predominant clinical symptoms of autoimmune disease awareness is necessary in order not to miss an underlying lymphoma. In this paper, we present a case that illustrates the difficulties encountered diagnosing malignant lymphoma when autoimmune phenomena dominate the clinical presentation.

A 53-year old woman was referred to our hospital for a second opinion. Seven months earlier, she had been admitted elsewhere because of intermittent fevers, rigors, night sweats, anorexia, weight loss, myalgia, arthralgia, and a maculopapular rash. Before this, there was no relevant medical history. On physical examination, no pathological lymph nodes, splenomegaly, or hepatomegaly was present. Laboratory investigation revealed a leukocytosis (34.7x $109 / 1)$ with $90 \%$ neutrophils, elevated C-reactive protein $(222 \mathrm{mg} / \mathrm{l})$, liver test abnormalities [alkaline phosphatase (168 U/l), aspartate transaminase (76 U/l), alanine transaminase (49 U/l)], elevated lactate dehydrogenase $(910 \mathrm{U} / \mathrm{l})$,

\footnotetext{
D. E. M. Geurts · W. J. F. M. van der Velden $(\bowtie)$ •

J. M. M. Raemaekers

Department of Haematology,

Radboud University Nijmegen Medical Centre,

P.O. Box 9101, 6500 HB Nijmegen, The Netherlands

e-mail: w.vandervelden@hemat.umcn.nl

K. M. Hebeda

Department of Pathology,

Radboud University Nijmegen Medical Centre,

P.O. Box 9101, 6500 HB Nijmegen, The Netherlands
}

and hyperferritinemia $(9,409 \mu \mathrm{mol} / \mathrm{l})$. Additional laboratory tests and serology did not show signs of rheumatoid diseases, sarcoidosis, viral, or bacterial infections (e.g., Epstein-Barr virus (EBV), cytomegalovirus, parvovirus, human immunodefficiency virus, mycoplasma, and borrelia). Computer tomography (CT) revealed two small lymph nodes in the left axilla, but a biopsy failed to acquire representative material. Eventually, the diagnosis of adult onset Still's disease (AOSD) was made because she met almost all criteria for this disease and no signs of a lymphoproliferative disease were found (Table 1) [3]. Treatment was started with prednisone (30 $\mathrm{mg}$ once daily), resulting in rapid clinical recovery.

August 2006, the patient was referred because lymphocytosis (absolute lymphocyte count, $5.7 \times 10^{9} / 1$ ) was noticed repeatedly during follow up, not accompanied by physical complaints or signs or cytopenias. After the prednisone was tapered and finally stopped, with immunophenotyping of peripheral blood (CD5, CD19, CD20, CD23, and Ig lambda positive), the diagnosis of a $\mathrm{B}$-cell chronic lymphatic leukemia (B-CLL) could be established, with RAI stage 0 , not necessitating therapy. A relation with the prior diagnosis of AOSD was not considered.

In November 2006, she was readmitted because she experienced a recurrence of the same complaints earlier resulting in the diagnosis of AOSD. Again, leukocytosis $\left(26.8 \times 10^{9} / 1\right)$ with predominantly neutrophils $(90 \%)$ and $8 \%$ lymphocytes was seen. A skin biopsy revealed aspecific dermatitis compatible with AOSD. Because of high fever causing headaches, treatment with naproxen and paracetamol was initiated without benefit. Bone marrow biopsy confirmed the earlier diagnosed B-CLL showing small foci of lymphocytic B cells with expression of CD5 and CD23 (Fig. 1a,b). CT now revealed generalized lymphadenopathy 
Table 1 Criteria for diagnosing AOSD

\section{Criteria}

Major criteria

Fever of $39^{\circ} \mathrm{C}$ or higher, lasting one week or longer

Arthralgia lasting two weeks or longer

Typical rash*

Leukocytosis $\left(10.000 / \mathrm{mm}^{2}\right.$ or greater) including 80 percent of granulocytes

Minor criteria

Sore throat

Lymphadenopathy** and/or splenomegaly

Liver dysfunction

Negative RF and negative ANA***

Exclusion criteria

Infection (especially sepsis and infectious mononucleosis)

Malignancies (especially malignant lymphoma)

Other rheumatoid diseases (especially polyarteritis nodosa, and rheumatoid vasculitis with extraarticular features)

Classification of AOSD requires five or more criteria including two or more major criteria. Any disease listed under "exclusions" should be absent. All criteria are applicable only in absence of other clinical explanations.

${ }^{a}$ Macular or maculopapular non-pruritic salmon pink eruption usually appearing during fever

${ }^{\mathrm{b}}$ Lymphadenopathy is defined as recent development of significant lymph node swelling, and splenomegaly is confirmed on palpation or by echogram.

${ }^{\mathrm{c}}$ Rheuma factor (RF) is serum that must be negative by routine test for the detection of IgM RF, and serum antinuclear antibodies (ANA) must be negative by routine immunofluorescence test.

and splenomegaly. A surgical excision of an enlarged axillary lymph node was performed (Fig. 1c,d). Prominent paracortical expansion and infiltration of immunoblastic $\mathrm{T}$ lymphocytes (Fig. 1e) was seen admixed with large CD20, CD79a, IgA, and partially CD30-positive blastic B lymphocytes (Fig. 1f). The B lymphocytes were negative for $\mathrm{CD} 5, \mathrm{CD} 23$, and EBV. While clonal immunoglobulin gene rearrangements were detected, no clonal rearrangements of the T-cell receptor genes were found, finally confirming the diagnosis of diffuse large B-cell lymphoma (DLBCL). Within the clinical context, this was regarded as a transformation of the B-CLL, the so-called Richter's syndrome, following 10 months after the diagnosis of AOSD. After initiation of combination chemotherapy [cyclophosphamide, doxorubicin, vincristine, and prednisone (CHOP)] and rituximab, the patient recovered rapidly with normalization of the laboratory abnormalities and the lymphadenopathy. Since the completion of eight courses of $\mathrm{R}-\mathrm{CHOP}$, she remains in complete remission and without symptoms, now for more than 12 months.

To our knowledge, this is the first case report on Richter's syndrome in a patient with AOSD. Our patient probably had AOSD that was complicated by the develop- ment of a B-CLL, which later transformed to a DLBCL, because at presentation no evidence was found of lymphoma. The presence of a "subclinical" CLL at disease onset cannot be excluded since no immunoflowcytometry studies were performed on the blood at that time. Autoimmune diseases are known to precede the occurrence of malignant lymphoma [1], and there are, although few, reports on AOSD preceding lymphoma $[4,5]$. However, the time interval between the development of the Richter's syndrome and the initial diagnosis of AOSD in this case was rather short, which is 10 months, realizing that the time interval for lymphomas to occur in autoimmune disorders are rather in the range of many years.

On the contrary, CLL and DLBCL can present with autoimmune phenomena, but these consist predominantly of autoimmune cytopenias, such as autoimmune hemolytic anemia, idiopathic thrombocytopenia, and less common, pure red cell aplasia and neutropenia [6]. Polymorphous autoimmune phenomena do occur in lymphoma, and even reports on lymphoma masquerading AOSD exist, although extremely rare, but in most of these reports, T-cell lymphoma seem to be involved [7]. An association between B-CLL or DLBCL and polymorphous autoimmune phenomena such as AOSD has not yet been described. Theoretically, it might be possible for B-cell lymphomas to induce polymorphous autoimmune diseases. The role of B lymphocytes extends beyond producing antibodies, as they are antigen-presenting cells and producers of cytokines, activating and altering the function of $\mathrm{T}$ lymphocytes, and their role in the pathogenesis of autoimmune disorders mediated by $\mathrm{T}$ lymphocyte deregulation has been established [8]. In our patient, this course of events is however unlikely because symptoms of AOSD clearly preceded the diagnosis of lymphoma.

The diagnosis of AOSD often poses great difficulty because the disease can mimic many other diseases, such as systemic infections, vasculitis syndromes, and malignancies. Therefore, the final diagnosis must not only rely on the presence of several clinical features but also on firm exclusion of other diseases including lymphoproliferative disorders (Table 1) [3]. Furthermore, during follow-up, changing, new, or worsening clinical symptoms could result from lymphoma complicating AOSD creating again a diagnostic problem. On pathological examination, enlarged lymph nodes, as seen in $50-60 \%$ of AOSD, are difficult to differentiate from lymphoma on pathological examination, especially from T-cell lymphoma [9]. In our patient, during follow-up, the occurrence of lymphadenopathy created the same problem. The predominant infiltrate in her lymph node biopsy consisted of a mixture of immunoblastic B and $\mathrm{T}$ cells, as reported before in AOSD [10], with only a minority of B-cell blasts, first suggesting the differential diagnosis of a reactive lymph node or a T-cell lymphoma. 
Fig. 1 Pathological examination of bone marrow and lymph node of the patient. Histology of the CLL in the bone marrow biopsy $(\mathbf{a} \mathrm{HE} \times 40$, b CD23 $\times 40)$ and the transformation in the axillary lymph node $(\mathbf{c} \mathrm{HE} \times 20$, d $\mathrm{HE} \times 40$, e CD3 $\times 40$, f CD20 $\times 40$ ). The blastic B-cell proliferation (f) is accompanied by immunoblastic T-cells in the background (e)
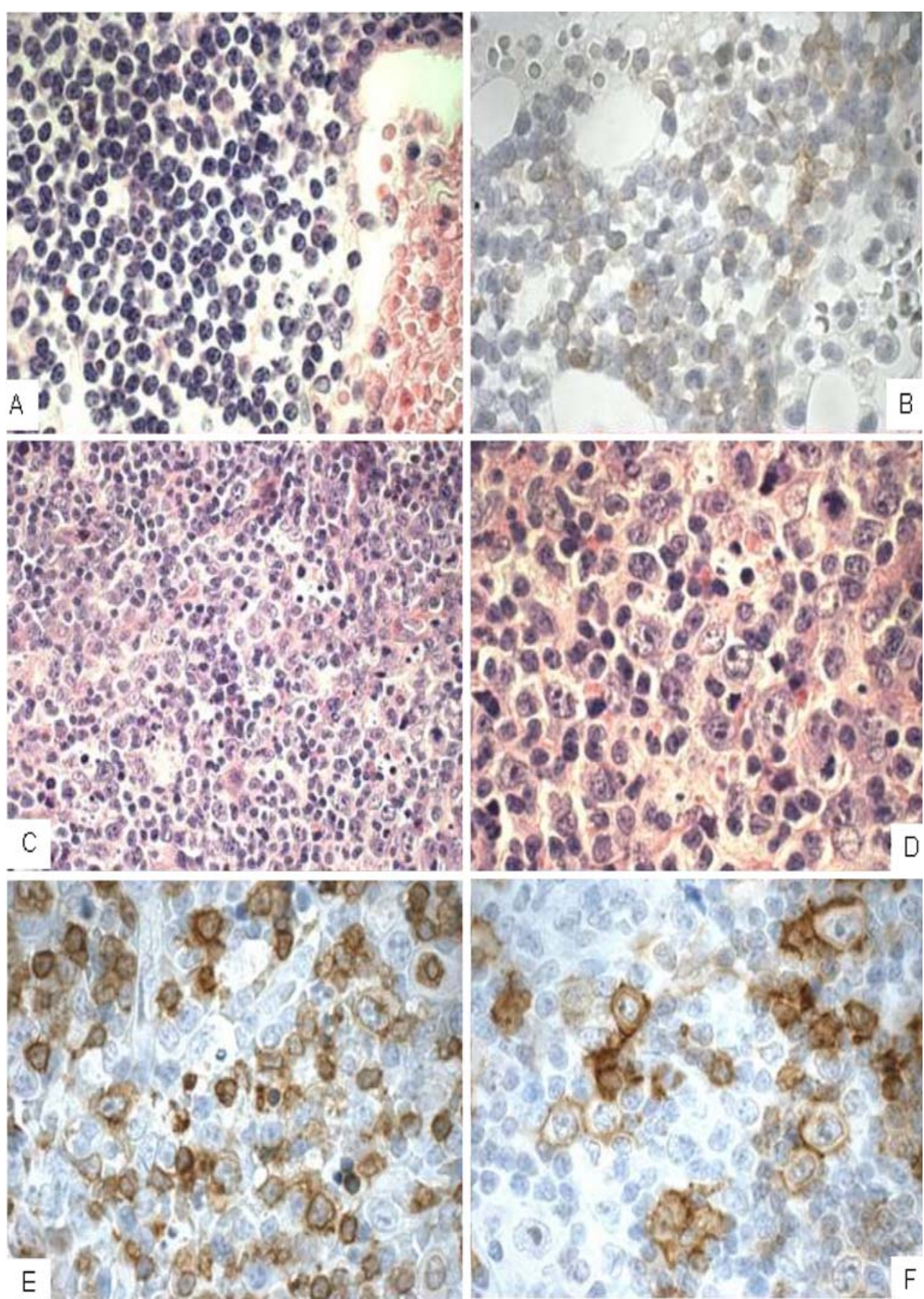

Determining an aberrant B-lymphocyte population by showing clonal immunoglobulin gene rearrangements was essential for the final diagnose of Richter's syndrome.

No strict recommendations exist on the extent of investigations that are required to exclude lymphoma before diagnosing AOSD. Furthermore, currently, no guidelines exist on screening for lymphoma in patients with autoimmune diseases in general, probably because the absolute incidence is low despite a substantial increased risk. In addition, it might be difficult to detect lymphoma in an early stage in patients with multiple aspecific symptoms, as mentioned earlier. Therefore, in practice, to avoid missing underlying lymphomas, extreme vigilance at diagnosis and during follow up is necessary. In case there is a changing or unusual clinical course, especially regarding lymphadenopathy, thorough investigations are obligatory including histopathology and immunophenotyping of blood, bone marrow, and lymph node biopsy. The determination of an aberrant or clonal lymphocyte population is of utmost importance in finally differentiating between autoimmune diseases and lymphoma. 
Open Access This article is distributed under the terms of the Creative Commons Attribution Noncommercial License which permits any noncommercial use, distribution, and reproduction in any medium, provided the original author(s) and source are credited.

\section{References}

1. Smedby KE, Baecklund E, Askling J (2006) Malignant lymphomas in autoimmunity and inflammation: a review of risks, risk factors, and lymphoma characteristics. Cancer Epidemiol Biomarkers Prev 15(11):2069-2077

2. Varoczy L, Gergely L, Zeher M, Szegedi G, Illes A (2002) Malignant lymphoma-associated autoimmune diseases-a descriptive epidemiological study. Rheumatol Int 22(6):233-237

3. Masson C, Le Loet X, Liote F, Dubost JJ, Boissier MC, PerrouxGoumy L, Bregeon C, Audran M (1996) Comparative study of 6 types of criteria in adult Still's disease. J Rheumatol 23(3):495-497

4. Sono H, Matsuo K, Miyazato H, Sakaguchi M, Matsuda M, Hamada K, Tatsumi Y, Maeda Y, Funauchi M, Kanamaru A
(2000) A case of adult-onset Still's disease complicated by nonHodgkin's lymphoma. Lupus 9(6):468-470

5. Trotta F, Dovigo L, Scapoli G, Cavazzini L, Castoldi G (1993) Immunoblastic malignant lymphoma in adult onset Still's disease. J Rheumatol 20(10):1788-1792

6. Kipps TJ, Carson DA (1993) Autoantibodies in chronic lymphocytic leukemia and related systemic autoimmune diseases. Blood 81 (10):2475-87

7. Kawasaki T, Hirohata S, Hashimoto T, Miyashita H, Tanaka F (1995) T cell lymphoma masquerading as adult onset Still's disease. Clin Exp Rheumatol 13(3):410-411

8. Shlomchik MJ, Craft JE, Mamula MJ (2001) From T to B and back again: positive feedback in systemic autoimmune disease. Nat Rev Immunol 1(2):147-153

9. Reichert LJ, Keuning JJ, van BM, van Rijthoven AW (1992) Lymph node histology simulating T-cell lymphoma in adult-onset Still's disease. Ann Hematol 65(1):53-54

10. Quaini F, Manganelli P, Pileri S, Magnani G, Ferrari C, Delsignore R, Sabattini E, Olivetti G (1991) Immunohistological characterization of lymph nodes in two cases of adult onset Still's disease. J Rheumatol 18(9):1418-1423 